
\title{
25 Research Square \\ Targeted Inhibition of ROCK Specifically in the Polarization Interstitial Macrophages Suppresses Pulmonary Fibrosis
}

\section{Qingfang Li}

West China Hospital of Medicine: Sichuan University West China Hospital

\section{Yuan Cheng}

West China Hospital of Medicine: Sichuan University West China Hospital

\section{Zhenfei Bi}

West China Hospital of Medicine: Sichuan University West China Hospital

\section{Xuelei Ma}

West China Hospital of Medicine: Sichuan University West China Hospital

\section{Yuquan Wei}

West China Hospital of Medicine: Sichuan University West China Hospital

Xiawei Wei ( $\nabla$ xiaweiwei@scu.edu.cn )

West China Hospital of Medicine: Sichuan University West China Hospital

\section{Research}

Keywords: ROCK, idiopathic pulmonary fibrosis, macrophage, polarization, radiation induced pulmonary fibrosis, Rho/ROCK pathway, STAT3, Inhibitor Running title: ROCK in pulmonary fibrosis

Posted Date: August 31st, 2021

DOl: https://doi.org/10.21203/rs.3.rs-783533/v1

License: (c) (1) This work is licensed under a Creative Commons Attribution 4.0 International License. Read Full License 


\section{Abstract}

\section{Background}

Pulmonary fibrosis (PF) is a kind of progressive interstitial lung disease with no effective therapy. Rho/ROCK pathway has been confirmed to be activated in the process of PF in bleomycin-induced mice model in previous studies, which is involved in cell proliferation, tissue repair and regeneration.

Bleomycin-induced and radiation-induced pulmonary fibrosis mice models were used to explore the effects and mechanism of WXWHO265, a novel unselected ROCK inhibitor, in PF.

\section{Methods}

The bleomycin-induced mice models were constructed by intratracheal instillation. The radiation-induced mice model were established by bilateral thoracic irradiation. Intragastric administration was applied to the WXWHO265 treated groups one day after model established. Flow cytometry, qRT-PCR, HE staining, Masson staining, and immunohistochemistry $(\mathrm{IHC})$ analysis were used for further investigation.

\section{Results}

In both types of PF models, the fibrosis in the lung was severe and became worse as time progressed. The status of mice in WXWHO265 treated groups was better than control group (saline treated group). The proportion of M2 macrophages in the lung tissue of bleomycin-induced group significantly increased compared to control group. The proportion of M2 macrophage of day 28 was higher than day 14 and day 7 in both PF models. The proportions of M2 macrophage in WXWHO265 high dose group $(25 \mathrm{mg} / \mathrm{kg}$ ) and control group were statistically different $(p<0.05)$. The p-STAT3 in lung tissue was significantly decreased in the day 28 in PF models. In vitro macrophages polarization experiment, the macrophages transformed into M2 macrophages by IL-4 stimulating. The proportion of M2 macrophages decreased after treated with WXWHO265.

\section{Conclusions}

Inhibiting ROCK could significant ameliorate PF in mice model by regulating the polarization of interstitial macrophages. Furthermore, the results showed that ROCK regulated the polarization of M2 macrophages by mediating the phosphorylation of STAT3, which might be a potential target in treating PF.

\section{Background}

Pulmonary fibrosis (PF) is a kind of chronic, progressive, deadly interstitial lung disease (ILD) characterized by aberrant accumulation of extracellular matrix (ECM) and the proliferation of the fibroblast[1]. Its main pathological feature is lung tissue injury and repair[2]. It was estimated that 3-9 new patients were diagnosed per 100,000 every year in American, which was higher than Asian populations[3]. And the survival of idiopathic pulmonary fibrosis (IPF) patients was quite poor, with median survival of 3- 
3.5 years and the five year survival rate of approximately $20 \%[4-9]$. Elderly smoking male patients accounted for the majority of all the IPF patients[10].

Recently, radiotherapy is an important treatment method of carcinoma and sarcoma, especially in thoracic tumors such as lung cancer, breast cancer and thoracic esophageal carcinoma. The therapeutic efficacy of radiotherapy is positively correlated with the dose. However, the occurrence of radiationinduced lung injury is also positively correlated to the radiation dose and lung injury limited the application of radiation therapy. Once the radiation-induced lung injury is out of control, it will transformed into radiation induced lung fibrosis. The pathology of PF is quite complex and unclear and there is still no effective method to cure or reverse the process of PF except for lung transplantation[11], which is limited by high cost, lack of donors and severe adverse events. Patients with PF are usually treated with glucocorticoid and cytotoxic drugs, while these pharmacological treatments show limited efficacy[12]. Pirfenidone and nintedanib are two new drugs approved by the Food and Drug Administration for the treatment of IPF[13]. These drugs could ameliorate the symptoms of IPF patients but the effect was limited. Besides pirfenidone and nintedanib, there are some new inhibitors related to the process of PF being studied, such as TGF- $\beta 1$ inhibitors and plasminogen activator inhibitor-1 (PAl-1) [14].

Macrophages in lung played an important role in pulmonary fibrosis in both inflammatory phase and fibrosis phase[1, 15, 16]. Lung macrophages included alveolar macrophages and interstitial macrophages[17]. The role of alveolar macrophages were intensively researched in previous studies[18]. Interstitial macrophages were rarely reported in the pulmonary fibrosis and the role of interstitial macrophages in lung fibrosis was unclear. Recently, some studies showed that interstitial macrophages derived from monocytes from bone marrow made the critical difference[19].

In previous studies, Rho/ROCK signal pathway is involved in cell proliferation, tissue repair and renew function, even affecting the migration of fibrocytes[20]. Rho protein includes more than 20 subtypes, among which RhoA, RhoB and RhoC are well-investigated[21]. GTP/GDP binding domain in Rho protein catalyzes the hydrolysis of GTP, which inactivates Rho protein. There are various proteins in the upstream and downstream of the Rho/ROCK pathway. ROCK is the first effect protein found in the downstream[22]. There are two subtypes of ROCK, ROCK1 and ROCK2. ROCK1 mainly expresses in lung, liver and kidney, while ROCK2 mainly expresses in heart and brain[23, 24]. Rho/ROCK pathway plays an important role in inflammation of lung fibrosis through mediating the release of inflammation factor, including TNF-a, TGF$\beta 1$ and IL-4, and affecting polarization of immune cells[25, 26]. Studies have found Rho/ROCK signal pathway was closely related to oxidative stress[27-29]. Researchers found that ROCK inhibitors suppressed the oxidative stress in lung injury induced by hypoxia[30]. Activated Rho/ROCK affects the phosphorylation of MLC, regulating the migrating of cells. ROCK inhibitor Y27632 reduced the fibrosis in liver and pancreas through inhibiting the migration of cells. Y27632 also ameliorate lung fibrosis by suppressing fibrocyte migrating in bleomycin-induced lung fibrosis model[31]. Rho/Rock pathway can promote the fibrocyte transferring to myofibroblast by inducing epithelial-mesenchymal transition. Rho/ROCK signal pathway promotes the fibrocyte transfer to myofibroblast which secreted collagen. In 
hyperoxia-induced lung fibrosis model, ROCK inhibitor suppressed formation of myofibroblast by reducing the production of a-SMA[32]. ROCK inhibitor fasudil ameliorate the lung fibrosis by inhibiting of epithelial-mesenchymal transition. In bleomycin-induced lung fibrosis, fasudil decreased the expression of a-SMA in lung tissue[33]. In IPF patients, p53, p21, Bax and Caspase-3 were obviously up-regulated. In mice PF, the expression of Bax and Caspase-3 were inhibited by the ROCK inhibitor[34]. However, ROCK inhibitors in radiation induced pulmonary fibrosis has not been investigated yet. Researchers considered that Rho/ROCK pathway could be activated by radiation[35], but no ROCK inhibitors were used in radiation induced pulmonary fibrosis before.

WXWHO265 is a new kind unselective ROCK inhibitor, which has not been reported before. In previous studies, both ROCK1 and ROCK2 played an important role in the process of pulmonary fibrosis. So we considered that the unselective inhibitor is more effective. Based on our previous studies, the WXWHO265 has many advantages, such as lose dose, safety and low side effects. To explore the mechanism of WXWHO265 in lung fibrosis, bleomycin and radiation-induced mice models were used in this study.

\section{Methods}

\subsection{Mice}

All mouse used in the experiment were breed according to the protocols approved by the Animal Care and Use Committee of Sichuan University. Inbred C57BL/6J mice were bred and maintained in the specific pathogen-free animal colony for mice model (Huafukang, Beijing).

\subsection{Bleomycin induced mice model and radiation induced mice model.}

10 week old male mice(6-7 mice in a group) were treated with $4 \mathrm{mg} / \mathrm{kg}$ bleomycin (Selleck, USA) to induce lung fibrosis model as the articles reported before. Bleomycin was administered through the intratracheal instillation to cause fibrosis[36, 37]. bleomycin was dissolved at $0.5 \%$ saline. The mice were divided into 5 groups, saline(control), bleomycin+saline, $10 \mathrm{mg} / \mathrm{kg}$ (WXWHO265)+bleomycin, 25mg/kg (WXWHO265)+ bleomycin, while in radiation induced group they were classified into control, radiation, $10 \mathrm{mg} / \mathrm{kg}$ (WXWHO265)+radiation, 25mg/kg (WXWHO265)+radiation. The mice were narcotized by intraperitoneal injection with $1 \%$ pentobarbital sodium. Whole thoraces of male C57BL/6J mice aged 10 weeks (6-7 mice in a group) were irradiated with 18Gy X-Gay (RS2000, 160kv, tube current $20 \mathrm{~mA}$, beam filter: $0.1 \mathrm{~mm} \mathrm{Cu}$ ) at dose rate of $3.116 \mathrm{~Gy} \cdot \mathrm{min}-1$. The drug was given after the second day model establishment by intragastric administration. Pulmonary fibrosis was further diagnosed by determination of pulmonary collagen (right lung) and histological examination (left lung). The care and use of animals was in accordance with the guidelines of the Institutional Animal Care and Use Committee of Sichuan University (Chengdu, Sichuan, China).

\subsection{Hydroxyproline}


The lung tissues obtained from the mice was placed in the ice and determined by alkaline hydrolysis. The concentrations of hydroxyproline were analyzed by hydroxyproline colorimetric assay kit (Jiancheng, Nanjing, China) and depend on the manufacturer's procedure.

\subsection{Histological examination}

Lung samples were fixed in 10\% formalin buffer (Wako Pure Chemical Industries, Ltd., Osaka, Japan) for histological examination. Paraffin sections ( $3.5 \mu \mathrm{m}$-thick) were cut from fixed lungs, stained with hematoxylin and eosin (HE) to assess gross morphology and Masson's trichrome stain to visualize collagen deposition, and examined by microscopy. The degree and grade of pulmonary fibrosis were decided by histological examination and hydroxyproline. The right lung was fixed in $4 \%$ paraformaldehyde. The tissue was treated at least 48 hour. Then the tissue was dewaxed with xylene for 60 minutes for 3 times and hydrated in a series of graded concentrations of ethanol (100\% ethanol for 5 min, $95 \%$ ethanol for $1 \mathrm{~min}, 80 \%$ ethanol for $5 \mathrm{~min}, 75 \%$ ethanol for $5 \mathrm{~min}$ and distilled water for $2 \mathrm{~min}$ ). After embedded in paraffin, the tissues were cut to $4 \mu \mathrm{m}$ thickness. Paraffin sections ( $4 \mu \mathrm{m}$-thick) were stained with HE and Masson's trichrome. Each lung of the mice was obtained at least 5 areas(200x) for assessing the severity of interstitial fibrosis by two pathologists by Szapiel[38] and Ashcroft scoring system[39]. For IHC, primary antibodies against ROCK1, ROCK2, Collagen-1, a-SMA were used as instruments. Each lung of the mice was obtained at least 5 areas (200x) for analysis and the analysis was performed by Image pro-plus 7.0.

\subsection{Quantitative Real-time Reverse Transcription-PCR (qRT-PCR)}

The lung tissue samples were dealt with total RNA isolation kit (Foregene, Chengdu, China). The total RNA were deleted with gDNA and transverse into cDNA with the Prime Script RT reagent kit (TaKaRa, Japan). The SYBR Green Real-Time PCR master mixes (Bio-Rad,USA) were used in qRT-PCR by CFX Connect Real-Time PCR system (Bio-Rad, USA). The primers were listed in supplementary Table 1.

\subsection{Cell culture}

Bone marrow-derived macrophages (BMDMs) were harvested from bone of lower limbs of adult C57BL/6J mice. The cells were plated at $10-\mathrm{cm}$ dishes with DMEM medium including $20 \mathrm{ng} / \mathrm{mL}$ of mouse MCSF-1 (R\&D and Prepotech) for 5-7days. Then the BMDM was stimulated with $20 \mathrm{ng} / \mathrm{mL}$ Mouse IL-4 (Prepotech) in vitro. After 6 hour incubation, the WXWHO265 was added to the medium. The flow cytomery and qRT-PCR were performed after 48 hour. The humidified incubator were with $95 \%$ air and $5 \%$ $\mathrm{CO}_{2}$ at $37^{\circ} \mathrm{C}$.

\subsection{Western blot}

Western blot performed as the previously articles procedure. About $20 \mathrm{mg}$ lung tissue were added into $1 \mathrm{ml}$ RIPA lysis buffer with $1 \mathrm{mM}$ phenylmethylsulphonyl fluoride. The lysates were collected and centrifuged at $13000 \mathrm{rpm}$ for10 minute. Each samples contain same amounts of proteins $(30 \mu \mathrm{g})$ and were mixed with 
buffer by heating $95 \mathrm{C}$ for 10 minute. The samples were resolved on $12.5 \%$ Bis-Tris Gel (Invitrogen Nupage Novex), transferred to $45 \mathrm{um}$ PVDF membrane and then incubated in TBST buffer $(150 \mathrm{mmol} / \mathrm{L} \mathrm{NaCl}, 20$ $\mathrm{mmol} / \mathrm{L}$ Tris-HCl, $0.02 \%$ Tween 20 [pH 7.4]) containing 5\% non-fat milk. Antibodies against ROCK1, ROCK2, STAT3, P-STAT3, $\beta$-tumblin were examined. Then the PVDF membrane was probed with antirabbit or mouse IgG/HPR (1:5000-10000) respectively, after this the membrane were visualized by ECL.

\subsection{Flow cytometry}

After sacrificed, the lung tissue obtained from the mice were minced by scissor and digested by the mixture of collagen- 1 and collagen-4, respectively. The suspensions cells were filtered via $70-\mu \mathrm{m}$ nylon mesh. The cells were centrifuged at $1500 \mathrm{rpm}$ for $5 \mathrm{~min}$ and washed by PBS for 3 times. Cells in blood from mice were directly isolated and washed by PBS for 3 times. The cells for flow cytometry was stained with antibody for 30 minutes in $4^{\circ} \mathrm{C}$. The macrophages were stained by the following antibodies: Percific blue-conjugated anti-mouse CD11b (1:100; BD Biosciences), PE-conjugated anti-mouse CD206 (1:100; BD Biosciences), APC-conjugated anti-mouse F4/80 (1:100; BD Biosciences), PerCP-CY5..5-conjugated antimouse CD45 (1:100; BD Biosciences), and FITC-conjugated anti-mouse CD11C (1:100; BD Biosciences). The monocytes in blood were stained by the following antibodies: PE-conjugated anti-mouse Ly6G (1:100; BD Biosciences), APC-conjugated anti-mouse CD11b (1:100; BD Biosciences), PerCP-CY5.5conjugated anti-mouse CD45 (1:100; BD Biosciences), and FITC-conjugated anti-mouse Ly6G (1:100; BD Biosciences). Cells were performed by flow cytometry from FlowJo (Tree Star, Inc.).

\subsection{Reagents and antibodies}

WXWHO265 (an unselective ROCK inhibitor) was obtained from Yaomingkangde (Nanjin, China). In vivo, WXWHO265 was prepared in 5\% DMSO and $95 \% \mathrm{ddH}_{2} \mathrm{O}$. Furthermore, the concentration of DMSO is limited in $0.1 \%$ in vitro. Antibodies were purchased from the following manufacturers: Anti-STAT3 (Cat\# 4904), anti-pSTAT3 (Y705) (Cat\# 9145) were obtained from Cell Signaling Technology (Cambridge, MA, USA). Anti-a-SMA (Cat\# MAB1420) were purchased from R\&D (USA). Anti-ROCK1 (Cat\# ab45171), antiROCK2 (Cat\# ab71598) and anti-Collagen-1 (Cat\# ab434710) were obtained from Abcam (Cambridge, UK). Anti- $\beta$-tumlin was purchased from Huaan Technology (Beijing, China).

\subsection{Statistical analysis}

All the data are presented as the mean \pm standard error of mean (SEM). Statistical analysis was performed by GraphPad Prism 5.0 software (GraphPad, San Diego, CA, USA). Unpaired T test was used to exam the differences in various condition. One-way ANOVA was used to perform statistical analysis of multiple groups. All the Statistical analysis was performed by Graphpad. $\mathrm{P}<0.05$ was considered as significant.

\section{Results}

3.1 WXWHO265 decreased the lung weight, lung index and hydroxyproline content of bleomycin-induced mice model 
The structure of the ROCK inhibitor WXWHO265 was shown in Figure 1A. Bleomycin-induced pulmonary fibrosis model was performed to evaluate the effect of WXWHO265 in mice (Figure 1B). Mice was given WXWHO265 by intragastric administration every day after modeling and then euthanized on day 7, 14 and 28 for analysis of fibrosis. The wet weight of lung in bleomycin treated model group was significant increased compared to control group (Figure 1C). Control group were the mice treated with saline by intratracheal instillation administration. Lung wet weight of high dose WXWHO265 treated group declined compared to bleomycin+saline group in each three point. Lung index was calculated by wet weight of lung dividing mice weight. The lung index in bleomycin-induced model group were significantly higher than that in saline group (Figure 1D). In day 7, the lung index were not significantly different between the low dose and high dose WXWHO265 treated group. In WXWHO265 treated groups, lung index of low dose $(10 \mathrm{mg} / \mathrm{kg})$ and high dose group $(25 \mathrm{mg} / \mathrm{kg}$ ) were significantly reversed compared with the fibrosis model group at day 14 and 28 . We further detected the content of hydroxyproline in lung to determine the accumulation of collagen at three time points (Figure 1E). In bleomycin model group, the content of hydroxyproline in lung was significantly increased comparing to saline treated group as shown in Figure 1. In two WXWHO265 treated groups, the content of hydroxyproline was lower than that of bleomycin treated group. In high dose group, the hydroxyproline content was lower than that in low dose group and with significant difference.

\subsection{WXWHO265 alleviated the pathological changes in PF in vivo.}

The histological injury and collagen deposition of lung tissue induced by bleomycin was assessed by $\mathrm{H} \& \mathrm{E}$ and masson staining, relatively. In saline treated group, it was observed that no obvious inflammation and fibrosis in the lung tissue of mice. The histological changes at day 7 and 28 were shown in Figure 1F.

After bleomycin intratracheal injection, the structure of alveolar was disordered and the airway wall was significantly thicken, in which the collagen deposited (Figure 1F). Collagen expression in model group significantly increased comparing to saline treated group after day 7 and increased progressively in day 14 and 28. There was obviously alveolar inflammation existing in lung tissue and amount of leukocytes infiltration in alveoli septum at day 7 . At day 14 , alveolar inflammation was severer than before and the alveolar space got thicken. It was obvious that fibroblasts and matrix were accumulated in lung tissue. There were more fibroblasts and matrix existing in lung tissue at day 28. A number of lymphocytes were found in lung tissue. In day 28 , the collagen deposition in model group was quite severe. In WXWHO265 treated group, collagen deposition decreased comparing with model group and solution group. The effect of WXWHO265 against bleomycin-induced inflammation and fibrosis was examined on day 7, 14 and 28 after bleomycin-induced model establishment. Although fibrotic lesions were observed in the WXWHO265 treated group, both the extent and intensity of the lesions were less than those of the bleomycin treated group. Lung sections in WXWHO265 treated group presented moderate inflammatory cells infiltration, relatively normal alveolar structure, slightly thicken alveolar wall and few lymphocytes and plasma cells. Significant morphological differences were observed in the two WXWHO265 treated groups either. The grade of alveolitis and inflammation was evaluated by the by the Szapiel score in Figure 1G-I. 
WXWHO265 of 10 and $25 \mathrm{mg} / \mathrm{kg}$ were able to attenuate the lung inflammation. The severity of lung fibrosis was systematically assessed by Ashcroft score (Figure 1J-L). The Ashcroft score of blemomycin treated mice significantly increased compared with saline treated mice. Administration of unselected ROCK inhibitor WXWHO265 resulted in a decrease in Ashcroft score.

\subsection{WXWHO265 reduced the expression of ROCK1, ROCK2, a-SMA and Collagen-1 in lung tissue of pulmonary fibrosis mice model}

The expression of ROCK1 and ROCK2 increased in bleomycin model group and high dose of WXWHO265 could significantly decrease the ROCK1 and ROCK2 expression (Figure 2A, B, C). The expression of a-SMA is considered as a typical biomarker of fibroblast transferring to myofibroblast, which could be observed in the alveolar and interstitial space of the lung. Based on analysis of IHC results, a-SMA expression in bleomycin-induced mice model was significantly higher than that of saline group and there were no significant difference between the two groups in day 7, 14 and 28 (Figure 2A, D). In WXWHO265 treated groups, the expression of a-SMA in both dose group demonstrated a remarkable decrease compared with bleomycin treated group. Between the two WXWHO265 treated groups, there was no significant difference.

Collagen 1 was another vital factor in measuring ECM deposition in lung mesenchyme. In the pathological section in day 7, there was obvious collagen-1 deposition in alveolar and interstitial space of the lung (Figure 2A, E). The change of collagen-1 expression was similar to the a-SMA except for that in day 7. The collagen-1 expression in low dose WXWHO265 treated significant decrease. There was no significant difference between low dose of WXWHO265 group and bleomycin treated group.

\subsection{WXWHO265 regulated the expression of M1 and M2 macrophage relative markers in lung tissue.}

In previous studies, the proportion of M2 macrophage was up-regulated in bleomycin- induced pulmonary fibrosis mice model[40]. Arg-1 was one of the most important markers of M2 macrophage which could reflect the number and the proportion of M2 macrophage. In addition, M1 macrophage also played a crucial role in the process of pulmonary fibrosis. INOS was the specific marker in M1 macrophage, which could reflect the number and proportion of M1 macrophage. In bleomycin-induced mice model, the expression of Arg-1 in model group significantly increased in the three time point compared with saline group (Figure 2F). High dose of WXWHO265 could decrease the expression of Arg-1 in day 7. After the model establishment, the expression of iNOS significantly increased (Figure $2 \mathrm{G}$ ). In day 14, iNOS was down regulated by the WXWHO265. In the other two time point, the expression of iNOS were not different among the groups.

\subsection{WXWHO265 reduced the CD206, FIZZ-1 and YM-1 relative expression}

CD206, FIZZ-1 and YM-1 were the special markers of the M2 macrophage. Their expression reflected the quantitative change of $\mathrm{M} 2$ macrophages. So we detected the relative expression of these marker in lung tissue by qRT-PCR in mRNA level. After the model establishment, on day 28 , the pulmonary fibrosis 
presented in late stage. CD206, FIZZ-1 and YM-1 expression increased in bleomycin model treated group (Figure 3A-C). WXWHO265 could effectively reduce the expression of CD206, FIZZ-1 and YM-1 in a dosedependent manner.

\subsection{WXWHO265 decreased the polarization proportion of M2 macrophages in lung}

When M0 macrophages were stimulated by IL-4 or IL-10, they transformed into M2 macrophages. Macrophages in lung tissue include BMDMs and tissue-resident macrophages.

$\mathrm{CD} 45^{+} \mathrm{F} 4 / 80^{+} \mathrm{CD} 206^{+} \mathrm{CD} 11 \mathrm{C}^{-}$cells were considered as M2 macrophages deriving from BMDMs. At day 7 , the proportion of M2 macrophages did not significantly increase comparing to saline group (Figure 3D-E). In day 14, the proportion of M2 macrophage in bleomycin-induced model group were significantly upregulated (Figure $3 \mathrm{E}$ ). The proportion of M2 macrophage gradually increased as the pulmonary fibrosis aggravating.

After the bleomycin injected into intratracheal and lung exposed to radiation, lung fibrosis processes into two stage, early inflammatory stage and lately fibrotic stage. The infiltration of monocytes in blood usually significantly increases after inflammatory stimuli. They play an important role in pulmonary fibrosis. Interstitial macrophages in lung tissue are derived from monocytes. Monocytes from blood are marked as $C D 45^{+} \mathrm{CD} 11 \mathrm{~b}^{+}$Ly $6 \mathrm{G}-\mathrm{Ly} 6 \mathrm{C}^{\text {high }}$. The monocytes were detected by flow cytometry at day 7 (Figure $3 F, G)$. The proportion of monocytes in blood of the bleomycin model increased to twice that of saline group. WXWHO265 could reduce the proportion of monocytes which was close to that of control group.

\subsection{WXWHO265 decreased the PF in radiation-induced mice model}

In radiation-induced mice model, the change of hydroxyproline content, lung weight and lung index were similar to that the bleomycin induced mice model (Figure 4A-D). The histological analysis of HE in radiation treated group showed significant inflammation injury, necrosis and pneumonitis at day 7 , whereas Masson staining show no obvious blue fiber deposition (Figure 4E-K). In week 12 and 16, extensive collagen was accumulated in irradiated group. In WXWHO265 treated groups, the inflammation and blue fiber deposition was decreased. The expression of ROCK1 and ROCK2 increased in irradiated model group and high dose WXWHO265 could significantly decrease the expression of ROCK 1 and ROCK2 (Figure 5A, B). In radiation mice model, after treated with low dose WXWHO265, the expression of a-SMA was not decreased at day 7 (Figure 5C). In week 12 and 16 (the middle and late stage of lung fibrosis), WXWHO265 significant inhibited the deposition of a-SMA. The change of collagen-1 in radiation-induced model was shown in Figure 5D, which was similar to bleomycin mice model.

Unlike bleomycin treated model, the expression of Arg-1 in irradiated mice model was significantly down regulated in high dose WXWHO265 treated group, while low dose WXWHO265 could not reduce the Arg-1 expression (Figure 5E). After model established, the expression of iNOS was up-regulated. WXWHO265 could not decrease the expression of iNOS (Figure 5F). In the radiation-induced mice model, the expression of CD206, FIZZ-1 and YM-1 were detected at week 16, which showed similar change with bleomycin model (Figure 6A-C). In radiation-induced mice model, the proportion of M2 macrophage 
significant increased at day 7, week 12 and week 16. In both dose of WXWHO265 treated group, the polarization of M2 macrophage was significantly reduced except for the low dose WXWHO265 treated at day 7 (Figure 6D). The proportion of monocytes in blood were detected by flow cytometry at day 7 (Figure $6 \mathrm{E})$. The infiltration of monocytes in the blood of radiation model increased to three times about that of control group. The proportion of monocytes was significantly down-regulated by high dose of WXWHO265.

\subsection{WXWHO265 inhibited the polarization of M2 in pulmonary fibrosis}

To check whether inhibiting ROCK influenced the polarization of macrophage, we assessed the expression of p-STAT3, STAT3, ROCK1 and ROCK2 (Figure 7). We discovered that the expression of ROCK1 and ROCK2 proteins was significantly increased after modeling. After treated with the inhibitor WXWHO265, these proteins were decreased and the protein decreased more in high dose group. The phosphorylation level of STAT3 was up-regulated after modeling and significantly reduced by WXWHO265. In qRT-PCR analysis, the expression of IL-10 was up-regulated after bleomycin modeling (Figure 7).

\subsection{WXWHO265 suppressed M2 polarization in vitro}

Based on the prior experiment, M2 macrophage of interstitial macrophage in lung played an important role in the process of PF. In mice treated with WXWHO265, the proportion of M2 macrophages was downregulated. To test the role of ROCK in the polarization of M2 macrophage of interstitial macrophage, we extracted BMDM cells from mice in vitro. Firstly, we treated the BMDMs with IL-4 for 2 hours. Then, we treated the cells with WXWHO265 for 48h (Figure 7E). The result showed that polarization of M2 macrophages was inhibited by WXWHO265 in a dose-dependent manner (Figure 7F). The expression of M2 macrophage related protein, Arg-1 and CD206, were also down-regulated (Figure 7G-H).

\section{Discussion}

In present study, the outcomes demonstrated that a nonselective ROCK inhibitor WXWHO265, effectively prevented radiation and bleomycin-induced PF by inhibiting the polarization of M2 macrophages of interstitial macrophages in lung. The analysis of the wet weight of lung, lung index, hydroxyproline content, the express of a-SMA and collagen-1 and pathological section were performed to evaluate the efficacy of WXWHO265 for PF. Then the expression of Arg-1 and iNOS was tested by IHC. The results showed that M2 macrophage were significantly related to the process of PF and regulated by ROCK inhibitor. In qRT-PCR analysis, the expression of CD206, FIZZ-1 and YM-1 were down-regulated by WXWHO265. The M2 macrophages of interstitial macrophages in lung were increased after modeling and they were reduced after WXWHO265 treated. The interstitial macrophages could be derived from monocytes in blood. The monocytes in blood was also increased after modeling and they were reduced after WXWHO265 treated. In vitro, the BMDMs were stimulated by IL-4 and the polarization of M2 macrophages was inhibited by WXWHO265 with dose-effect relationship. And the expressions of Arg-1 
and CD206 were presented the similar trends, which indicated that ROCK inhibitor played an important role in PF.

Rho/ROCK pathway was activated in the process of bleomycin induced PF in mouse model reported in previous studies[41]. Rho/ROCK pathway takes part in the process of cell proliferation, tissue repair and regeneration[42]. Besides, it can also mediate the migration of fibroblasts[43]. There are lots of proteins in its upstream promoters or downstream promoters. ROCK is the first protein found regulated by Rho and it has two subtypes, ROCK1 and ROCK2[44]. ROCK1 is mainly expressed in the lung, liver, kidney. ROCK2 is mainly expressed in the heart and brain[45]. Studies has found that ROCK1 is related to M1 macrophages and ROCK2 is correlated to M2[46]. The downstream phosphorylation substrate includes LIM kinases (LIMK), myosin light chain (MLC) and myosin phosphatase target subunit-1 (MYPT-1). These substrates can mediate the contractility of actin. In previous studies, the researchers found that ROCK1- or ROCK2haploinsufficient mice could protect mice from bleomycin-induced PF. ROCK1 and ROCK2 haploinsufficient synchronously (ROCK $1^{+/-} 2^{+/-}$, ROCK $1^{+/-} 2^{+/}$) made greater contributions to protect against the bleomycin induced pulmonary fibrosis than ROCK1- or ROCK2- haploinsufficient[47]. In this study, we investigated a nonselective ROCK inhibitor, WXWHO265, on suppressing the lung fibrosis induced by bleomycin. The results showed WXWHO265 could significantly ameliorate the pulmonary fibrosis by regulating the polarization of macrophages in bleomycin or radiation-induced model.

The infiltration of inflammatory cells were increased in air spaces and lung interstitium after intratracheal instillation of bleomycin. The expressions of cytokines, chemokines, reactive oxygen species, and proteases in PF resulted in proliferation of myofibroblast and deposition of collagen. Macrophages, neutrophils and monocytes play an important role in the process of PF, especially macrophages. Macrophages in lung are divided into alveolar macrophages (AMs) and interstitial macrophages (IMs) based on its location [48, 49]. AMs usually resident in alveolar from embryonic period and they can selfrenew rather than transformed from monocyte in peripheral blood system. Some studies reported that AMs could also originate from monocytes from peripheral circulating. It is usually considered that recruitment of AMs in lung is mainly associated with inflammatory phase, immune signaling and arginine metabolism, while AMs resident in lung mainly take part in proliferation, and fatty acid metabolism pathways[50,51]. Previous study reported that the deletion of tissue-resident alveolar macrophage did not affect the severity of pulmonary fibrosis, while deleting the monocyte-derived alveolar macrophages could ameliorate PF[50]. However IMs has not been well studied in the pathology of PF. IMs derived from BMDMs. Some studies reported that they could also derive from yolk-sac macrophages[52]. Compared with IMs, CD11C is a specific surface marker expressed on the AM[53, 54].

M1 (classically activated macrophages) and M2 (alternatively activated macrophages) macrophages were another subtypes of macrophage. M1 macrophages play an essential role in tissue inflammation and repair[55]. M2 macrophages are responsible to the relief of tissue repair and deposition of ECM[56]. M1 macrophages are responsible for wound healing after alveolar epithelial injury, while M2 macrophages are designated to resolve wound healing processes or terminate inflammatory responses in the lung[57]. In PF patients, the expression of M2 markers were observed significantly increased[58]. It 
was reported that at the late stage of PF, M2 macrophage of IM was significant increased. CD206 is the first known alternative macrophage activation surface marker, which is increased in IPF patients[40]. Interestingly, the activation of CD206 was also related to the extra cellar matrix of cells such as collagen[59]. In our study, the relative expression of CD206, FIZZ-1 and YM-1 were down-regulated after ROCK inhibitor treated. Our findings confirmed that M2 macrophages population played an important role in the process of lung fibrosis. The results demonstrated that M2 macrophages of IMs took part in the process of PF. And the polarization of M2 macrophage was regulated by ROCK. In vitro, the similar phenomenon was observed.

When the ly $6 \mathrm{C}^{\text {high }}$ monocytes were stimulated by some specific cytokines, the cells infiltrated into lung tissue and transferred into IMs. The study of Gibbons et al. demonstrated that the increase of Ly6C ${ }^{\text {high }}$ "inflammatory" monocytes facilitated the progression of the fibrotic phase in PF[60-62]. It was also speculated that Ly6 $\mathrm{C}^{\text {high }}$ monocytes were the precursors of BMDMs[63]. In our study, the Ly6 $\mathrm{C}^{\text {high }}$ monocytes significantly increased after modeling and the proportion of monocytes was suppressed by the WXWHO265 at the early stage. Ly6 $\mathrm{C}^{\text {high }}$ monocytes was not only a signal of inflammatory but also a potential therapeutic target in PF.

In previously studies, the level of IL-10 was significantly up-regulated in bleomycin induced mice model[64]. The soluble receptors of IL-10 binding with the inhibitor regulated the phosphorylation of two tyrosine residues[65]. Then the STAT3 was docked by the phosphorylated tyrosine residues. The function of STAT3 was not clear in pulmonary fibrosis and the phosphorylation of STAT3 definitely contribute to the deposition of collagen and release of IL-10 and IL-4[66]. Th2 cytokines, IL-4 and IL-10 could promote the polarization of M2 macrophage, which were correlated to the pathogenesis of lung fibrosis[67-69]. In our research, ROCK inhibitor could inhibit the phosphorylation of STAT3 via suppressing the expression of IL-10. It indicated that the polarization of M2 macrophages was associated with Rho/ROCK pathway by regulating the phosphorylation STAT3 via IL-10.

\section{Conclusion}

In conclusion, the results in our study demonstrated that nonselective ROCK inhibitor WXWHO265 significantly attenuated the bleomycin and radiation induced PF by inhibiting the polarization of M2 macrophage of IMs. It was the first time that the anti-fibrosis effect of ROCK inhibitor in radiation-induced PF model was reported. In vivo, the polarization of M2 macrophage was inhibited by ROCK inhibitor through reducing the phosphorylation of STAT3, ameliorating PF in mice models. In vitro, the ability of ROCK inhibitor to inhibit BMDM polarizing to M2 macrophage was confirmed in vitro. The results provide evidence that ROCK inhibitor, WXWHO265, is a potential target to prevent the development of PF.

\section{Abbreviations}

PF:Pulmonary fibrosis; IHC:Immunohistochemistry; ILD:Interstitial lung disease; ECM:extracellular matrix; IPF:Idiopathic pulmonary fibrosis; 6MWD:6-min walking test distance; FVC:Forced vital capacity; PAI- 
1:plasminogen activator inhibitor-1; HE:Hematoxylin and eosin; qRT-PCR:Quantitative Real-time Reverse Transcription-PCR; BMDM:Bone marrow-derived macrophage; SEM:Standard error of mean; LIMK:LIM kinases; MLC:Myosin light chain; MYPT-1:Myosin phosphatase target subunit-1; AM:Alveolar macrophages; IM: interstitial macrophage;

\section{Declarations}

Ethics approval and consent to participate Animal experiments were approved by the State Key Laboratory of Biotherapy Animal Care and Use Committee of Sichuan University, China.

Consent for publication Not applicable.

Availability of data and material The datasets in this study are available from the corresponding author on reasonable request.

Competing interests The authors declare that they have no competing interests.

Funding This work is supported by the National Natural Science Foundation Regional Innovation and Development (U19A2003), the National Major Scientific and Technological Special Project for "Significant New Drugs Development" (No. 2018ZX09733001, China), the Development Program of China (No. 2016YFA0201402), and by the Excellent Youth Foundation of Sichuan Scientific Committee Grant in China (No. 2019JDJQ008).

Author contributions QL, YW and XW conceived and designed the experiments. QL, YC, ZB and XM performed the experiments. QL and YC analyzed the data. ZB gave technical support and conceptual advice. QL,YC and YW wrote the manuscript. All authors commented on the manuscript.

Acknowledgments We would like to thank the colleagues from our group for their assistance.

\section{References}

1. Yanagihara T, Sato S, Upagupta C, Kolb M. What have we learned from basic science studies on idiopathic pulmonary fibrosis. Eur Respir Rev. 2019;28.

2. Strieter RM. What differentiates normal lung repair and fibrosis? Inflammation, resolution of repair, and fibrosis. Proc Am Thorac Soc. 2008. 5(3): 305-10.

3. Hutchinson J, Fogarty A, Hubbard R, McKeever T. Global incidence and mortality of idiopathic pulmonary fibrosis: a systematic review. Eur Respir J. 2015. 46(3): 795-806.

4. Spagnolo P, Sverzellati N, Rossi G, et al. Idiopathic pulmonary fibrosis: an update. Ann Med. 2015. 47(1): 15-27.

5. Agabiti N, Porretta MA, Bauleo L, et al. Idiopathic Pulmonary Fibrosis (IPF) incidence and prevalence in Italy. Sarcoidosis Vasc Diffuse Lung Dis. 2014. 31(3): 191-7. 
6. Ley B, Collard HR, King TE. Clinical course and prediction of survival in idiopathic pulmonary fibrosis. Am J Respir Crit Care Med. 2011. 183(4): 431-40.

7. Gribbin J, Hubbard RB, Le JI, Smith CJ, West J, Tata LJ. Incidence and mortality of idiopathic pulmonary fibrosis and sarcoidosis in the UK. Thorax. 2006. 61(11): 980-5.

8. Raghu G, Chen SY, Hou Q, Yeh WS, Collard HR. Incidence and prevalence of idiopathic pulmonary fibrosis in US adults 18-64 years old. Eur Respir J. 2016. 48(1): 179-86.

9. Navaratnam $\mathrm{V}$, Fleming $\mathrm{KM}$, West $\mathrm{J}$, et al. The rising incidence of idiopathic pulmonary fibrosis in the U.K. Thorax. 2011. 66(6): 462-7.

10. D Assayag JM, KA Johannson AUW. Patient gender bias on the diagnosis of idiopathic pulmonary fibrosis. Thorax. 2020 .

11. N Sharif MI. Familial idiopathic pulmonary fibrosis in two Pakistani siblings-case report and topic review. Science Postprint. 2015.

12. Juarez MM, Chan AL, Norris AG, Morrissey BM, Albertson TE. Acute exacerbation of idiopathic pulmonary fibrosis-a review of current and novel pharmacotherapies. J Thorac Dis. 2015. 7(3): 499519.

13. Raghu G, Selman M. Nintedanib and pirfenidone. New antifibrotic treatments indicated for idiopathic pulmonary fibrosis offer hopes and raises questions. Am J Respir Crit Care Med. 2015. 191(3): 252-4.

14. Somogyi V, Chaudhuri N, Torrisi SE, Kahn N, Müller V, Kreuter M. The therapy of idiopathic pulmonary fibrosis: what is next. Eur Respir Rev. 2019. 28(153).

15. Du S, Li C, Lu Y, Lei X, Zhang Y, Li S, Liu F, Chen Y, Weng D, Chen J. Dioscin Alleviates Crystalline Silica-Induced Pulmonary Inflammation and Fibrosis through Promoting Alveolar Macrophage Autophagy. Theranostics. 2019;9:1878-92.

16. Li G, Jin F, Du J, He Q, Yang B, Luo P. Macrophage-secreted TSLP and MMP9 promote bleomycininduced pulmonary fibrosis. Toxicol Appl Pharmacol. 2019;366:10-6.

17. Crowell RE, Heaphy E, Valdez YE, Mold C, Lehnert BE. Alveolar and interstitial macrophage populations in the murine lung. Exp Lung Res. 1992;18:435-46.

18. Larson-Casey JL, Deshane JS, Ryan AJ, Thannickal VJ, Carter AB. Macrophage Akt1 KinaseMediated Mitophagy Modulates Apoptosis Resistance and Pulmonary Fibrosis. Immunity. 2016;44:582-96.

19. Joshi N, Watanabe S, Verma R, Jablonski RP, Chen Cl, Cheresh P, Markov NS, Reyfman PA, McQuattiePimentel AC, Sichizya $L$, et al. A spatially restricted fibrotic niche in pulmonary fibrosis is sustained by M-CSF/M-CSFR signalling in monocyte-derived alveolar macrophages. Eur Respir J. 2020;55.

20. Tan HB, Zhong YS, Cheng Y, Shen X. Rho/ROCK pathway and neural regeneration: a potential therapeutic target for central nervous system and optic nerve damage. Int J Ophthalmol. 2011. 4(6): 652-7.

21. E Amin BND, SC Zhang LG. Rho-kinase: regulation,(dys) function, and inhibition. Biol Chem. 2013 . 
22. Harvey SA, Anderson SC, SundarRaj N. Downstream effects of ROCK signaling in cultured human corneal stromal cells: microarray analysis of gene expression. Invest Ophthalmol Vis Sci. 2004. 45(7): $2168-76$.

23. M Surma LW, Shi J. Rho kinase as a therapeutic target in cardiovascular disease. Future Cardiol. 2011.

24. Antoniu SA. Targeting RhoA/ROCK pathway in pulmonary arterial hypertension. Expert Opin Ther Targets. 2012.

25. Heukels P, Moor CC, der Thüsen JH v, Wijsenbeek MS, Kool M. Inflammation and immunity in IPF pathogenesis and treatment. Respir Med. 2019. 147: 79-91.

26. Liu J, Wada Y, Katsura M, et al. Rho-Associated Coiled-Coil Kinase (ROCK) in Molecular Regulation of Angiogenesis. Theranostics. 2018. 8(21): 6053-6069.

27. Y Zhang LD, S Wu PC, Zhao S. Atorvastatin attenuates involvement of RhoA/Rho-kinase pathway and NF-KB activation in hypoxic pulmonary hypertensive rats. Chin Med J (Engl). 2014 .

28. Kim JG, Sung HJ, Ok SH, et al. Calcium sensitization involved in dexmedetomidine-induced contraction of isolated rat aorta. Can J Physiol Pharmacol. 2011. 89(9): 681-9.

29. K Björling PDJ, Egebjerg K. Role of age, Rho-kinase 2 expression, and G protein-mediated signaling in the myogenic response in mouse small mesenteric arteries. Physiological reports. 2018 .

30. Xu W, Zhao Y, Zhang B, et al. Resveratrol attenuates hyperoxia-induced oxidative stress, inflammation and fibrosis and suppresses $\mathrm{Wnt} / \beta$-catenin signalling in lungs of neonatal rats. Clin Exp Pharmacol Physiol. 2015. 42(10): 1075-83.

31. J Ni ZD, W Han DK, Su Y. The role of RhoA and cytoskeleton in myofibroblast transformation in hyperoxic lung fibrosis. Free Radic Biol Med. 2013.

32. Y Shimizu KD, K lizuka TH. Contribution of small GTPase Rho and its target protein rock in a murine model of lung fibrosis. Am J Respir Crit Care Med. 2001.

33. C Jiang HH, J Liu YW, Lu Z. Fasudil, a rho-kinase inhibitor, attenuates bleomycin-induced pulmonary fibrosis in mice. Int J Mol Sci. 2012.

34. H Zhou YS, L Zhang WK. The RhoA/ROCK pathway mediates high glucose-induced cardiomyocyte apoptosis via oxidative stress, JNK, and p38MAPK pathways. Diabetes Metab Res Rev. 2018.

35. Haydont V, Bourgier C, Vozenin-Brotons MC. Rho/ROCK pathway as a molecular target for modulation of intestinal radiation-induced toxicity. $\mathrm{Br} J$ Radiol. 2007. $80 \mathrm{Spec}$ No 1: S32-40.

36. Bhandary YP, Shetty SK, Marudamuthu AS, Ji HL, Neuenschwander PF, Boggaram V, et al. Regulation of lung injury and fibrosis by p53-mediated changes in urokinase and plasminogen activator inhibitor-1. Am J Pathol 2013;183(1):131-143.

37. Gouda MM, Bhandary YP. Curcumin down-regulates IL-17A mediated p53-fibrinolytic system in bleomycin induced acute lung injury in vivo. J Cell Biochem 2018;119(9):7285-7299.

38. Szapiel SV, Elson NA, Fulmer JD, Hunninghake GW, Crystal RG. Bleomycin-induced interstitial pulmonary disease in the nude, athymic mouse. Am Rev Respir Dis. 1979. 120(4): 893-9. 
39. Ashcroft T, Simpson JM, Timbrell V. Simple method of estimating severity of pulmonary fibrosis on a numerical scale. J Clin Pathol. 1988. 41(4): 467-70.

40. Zhang L, Wang Y, Wu G, Xiong W, Gu W, Wang CY. Macrophages: friend or foe in idiopathic pulmonary fibrosis. Respir Res. 2018. 19(1): 170.

41. Riches DW, Backos DS, Redente EF. ROCK and Rho: Promising therapeutic targets to ameliorate pulmonary fibrosis. Am J Pathol. 2015. 185(4): 909-12.

42. Maharam E, Yaport M, Villanueva NL, et al. Rho/Rock signal transduction pathway is required for MSC tenogenic differentiation. Bone Res. 2015. 3: 15015.

43. Provenzano PP, Inman DR, Eliceiri KW, Trier SM, Keely PJ. Contact guidance mediated threedimensional cell migration is regulated by Rho/ROCK-dependent matrix reorganization. Biophys $\mathrm{J}$. 2008. 95(11): 5374-84.

44. L Julian MFO. Rho-associated coiled-coil containing kinases (ROCK) structure, regulation, and functions. Small GTPases. 2014 .

45. Hartmann S, Ridley AJ, Lutz S. The Function of Rho-Associated Kinases ROCK1 and ROCK2 in the Pathogenesis of Cardiovascular Disease. Front Pharmacol. 2015. 6: 276.

46. Zandi S, Nakao S, Chun KH, et al. ROCK-isoform-specific polarization of macrophages associated with age-related macular degeneration. Cell Rep. 2015. 10(7): 1173-86.

47. Knipe RS, Probst CK, Lagares D, et al. The Rho Kinase Isoforms ROCK1 and ROCK2 Each Contribute to the Development of Experimental Pulmonary Fibrosis. Am J Respir Cell Mol Biol. 2018. 58(4): 471481.

48. AV Misharin LM, Mutlu GM. Flow cytometric analysis of macrophages and dendritic cell subsets in the mouse lung. Am J Respir Cell Mol Biol. 2013.

49. Zaynagetdinov R, Sherrill TP, Kendall PL, et al. Identification of myeloid cell subsets in murine lungs using flow cytometry. Am J Respir Cell Mol Biol. 2013. 49(2): 180-9.

50. AV Misharin LM. Monocyte-derived alveolar macrophages drive lung fibrosis and persist in the lung over the life span. J Exp Med. 2017.

51. Mould KJ, Barthel L, Mohning MP, et al. Cell Origin Dictates Programming of Resident versus Recruited Macrophages during Acute Lung Injury. Am J Respir Cell Mol Biol. 2017. 57(3): 294-306.

52. CL Scott SH, Guilliams M. Mononuclear phagocytes of the intestine, the skin, and the lung. Immunol Rev. 2014 .

53. Guth AM, Janssen WJ, Bosio CM, Crouch EC, Henson PM, Dow SW. Lung environment determines unique phenotype of alveolar macrophages. Am J Physiol Lung Cell Mol Physiol. 2009. 296(6): L936-46.

54. R Zaynagetdinov TPS, Kendall PL. Identification of myeloid cell subsets in murine lungs using flow cytometry. Am J Respir Cell Mol Biol. 2013.

55. Atri C, Guerfali FZ, Laouini D. Role of Human Macrophage Polarization in Inflammation during Infectious Diseases. Int J Mol Sci. 2018. 19(6). 
56. TA Wynn KMV. Macrophages in tissue repair, regeneration, and fibrosis. Immunity. 2016.

57. Hesketh M, Sahin KB, West ZE, Murray RZ. Macrophage Phenotypes Regulate Scar Formation and Chronic Wound Healing. Int J Mol Sci. 2017. 18(7).

58. DV Pechkovsky AP, F Kollert KE. Alternatively activated alveolar macrophages in pulmonary fibrosismediator production and intracellular signal transduction. Clin Immunol. 2010 .

59. Allavena P, Chieppa M, Monti P, Piemonti L. From pattern recognition receptor to regulator of homeostasis: the double-faced macrophage mannose receptor. Crit Rev Immunol. 2004. 24(3): 17992.

60. Yona S, Jung S. Monocytes: subsets, origins, fates and functions. Curr Opin Hematol. 2010. 17(1): 53-9.

61. Tacke F, Randolph GJ. Migratory fate and differentiation of blood monocyte subsets. Immunobiology. 2006. 211(6-8): 609-18.

62. Lenzo JC, Turner AL, Cook AD, et al. Control of macrophage lineage populations by CSF-1 receptor and GM-CSF in homeostasis and inflammation. Immunol Cell Biol. 2012. 90(4): 429-40.

63. MA Gibbons ACM. Ly6Chi Monocytes Direct Alternatively Activated Profibrotic Macrophage Regulation of Lung Fibrosis. Am J Respir Crit Care Med. 2011.

64. L Sun MCL, Vannella KM. New concepts of IL-10-induced lung fibrosis: fibrocyte recruitment and M2 activation in a CCL2/CCR2 axis. Am J Physiol Lung Cell Mol Physiol. 2011.

65. Williams LM, Ricchetti G, Sarma U, Smallie T, Foxwell BM. Interleukin-10 suppression of myeloid cell activation-a continuing puzzle. Immunology. 2004. 113(3): 281-92.

66. Hu D, Wan L, Chen M, et al. Essential role of IL-10/STAT3 in chronic stress-induced immune suppression. Brain Behav Immun. 2014. 36: 118-27.

67. Huaux F, Liu T, McGarry B, Ullenbruch M, Phan SH. Dual roles of IL-4 in lung injury and fibrosis. J Immunol. 2003. 170(4): 2083-92.

68. Wallace WA, Ramage EA, Lamb D, Howie SE. A type 2 (Th2-like) pattern of immune response predominates in the pulmonary interstitium of patients with cryptogenic fibrosing alveolitis (CFA). Clin Exp Immunol. 1995. 101(3): 436-41.

69. SP Atamas VVY, Wise R. Production of type 2 cytokines by CD8+ lung cells is associated with greater decline in pulmonary function in patients with systemic sclerosis. Arthritis Rheum. 1999.

\section{Figures}




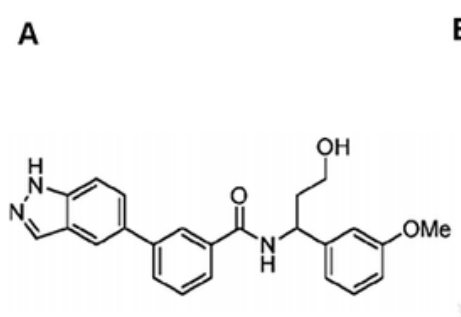

WXWHO265

B Intratracheal administration
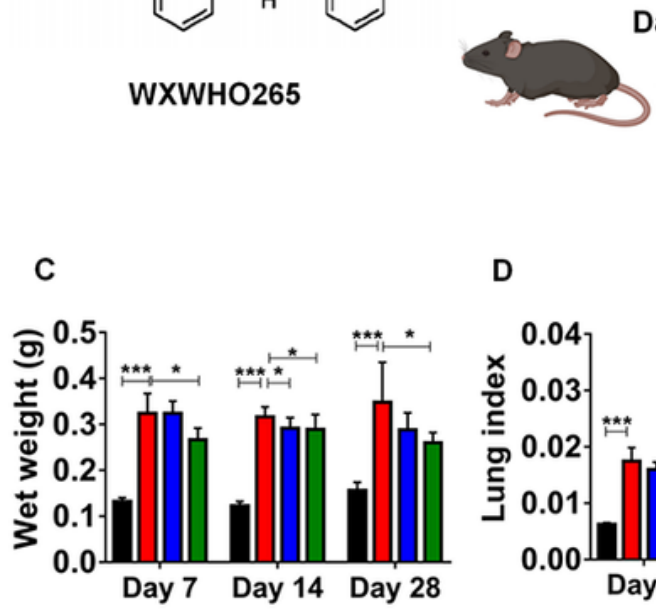

Day 0

WXWHO265 was adminstered via intragastric administration every day
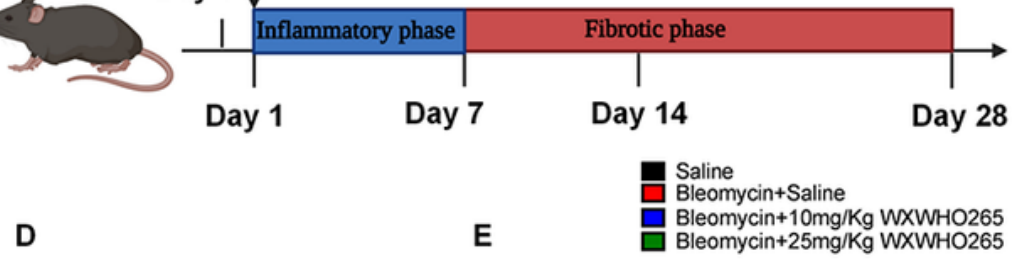

F

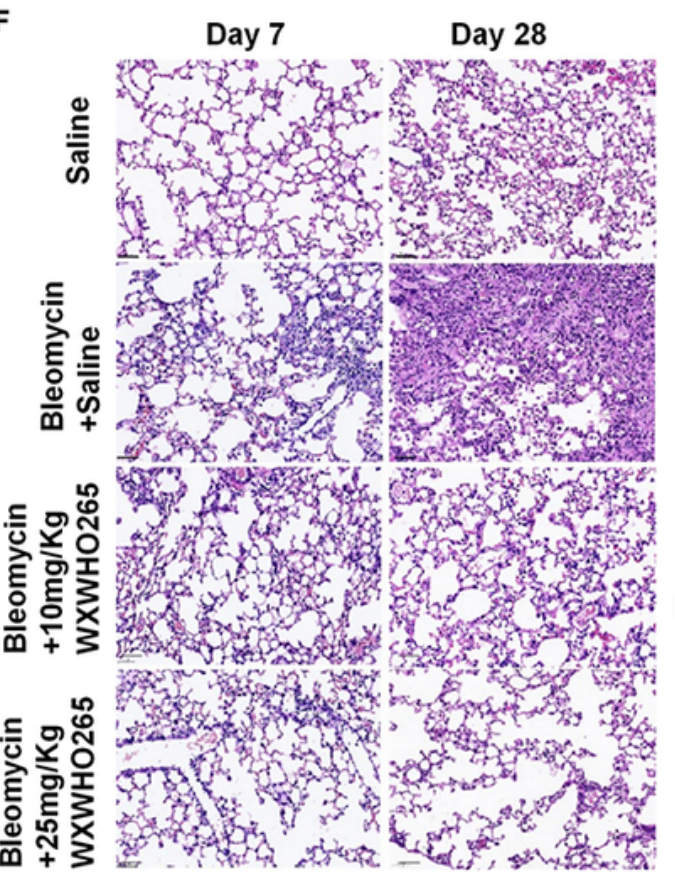

G
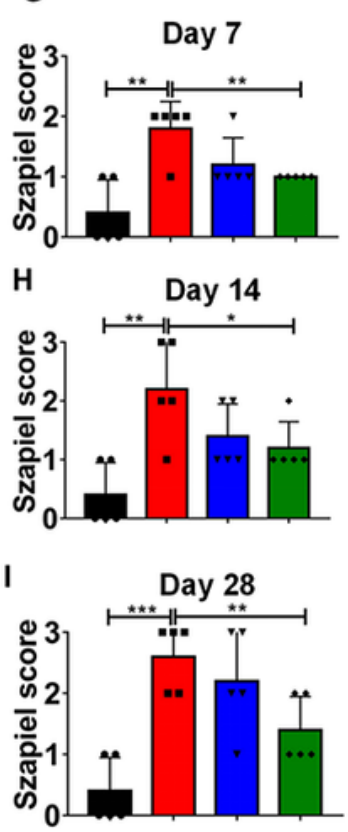

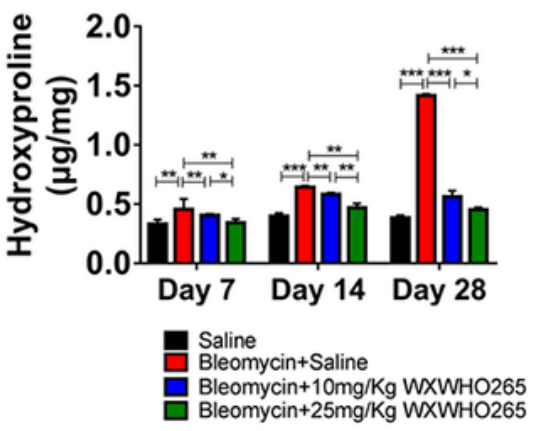

J
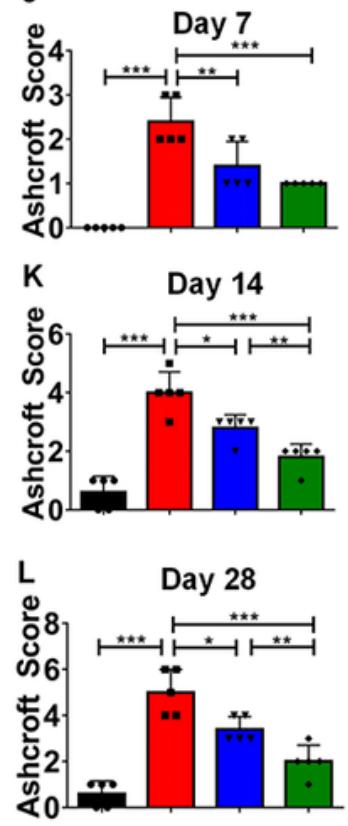

\section{Figure 1}

The treatment effects of ROCK inhibitor (WXWHO265) in bleomycin-induced lung fibrosis model. (A) The key structural features of the ROCK inhibitor (WXWHO265). (B)Schematic representation of the experimental protocol for model induction, treatment and endpoints in bleomycin-induced lung fibrosis mice model. For bleomycin induced mice model, $4 \mathrm{mg} / \mathrm{kg}$ bleomycin was injected by intratracheal instillation of C57BL/6 mice. n=6-7. At the day after modeling, the ROCK inhibitor WXWHO265 of 10 
$\mathrm{mg} / \mathrm{kg}$ and $25 \mathrm{mg} / \mathrm{kg}$ was administered by intragastric injection every day. (C-E) After intratracheal instillation of $4 \mathrm{mg} / \mathrm{kg}$ bleomycin or saline, the mice were treated with WXWHO265 or saline. The wet weight of lung (C), lung index (D), and hydroxyproline expression in lung (E) at day 7, day 14, day 28 are shown. Lung index was referred to lung/body weight ratio. (F) HE staining of mice lung tissues at day 7 and day 28. Representative pictures are shown, scale bar=50 $\mathrm{mm}$. (G-I) The inflammation changes in the lungs were quantified by a numerical inflammation score (Szapiel score) histopathologically. (J-L) The fibrotic changes in the lungs were quantified by a numerical fibrotic score (Ashcroft score) histopathologically. The data were analyzed using one-way ANOVA and shown as mean \pm SEM. ${ }^{*} p<0.05$, ${ }^{* \star} \mathrm{p}<0.01,{ }^{* \star *} \mathrm{p}<0.001$. 


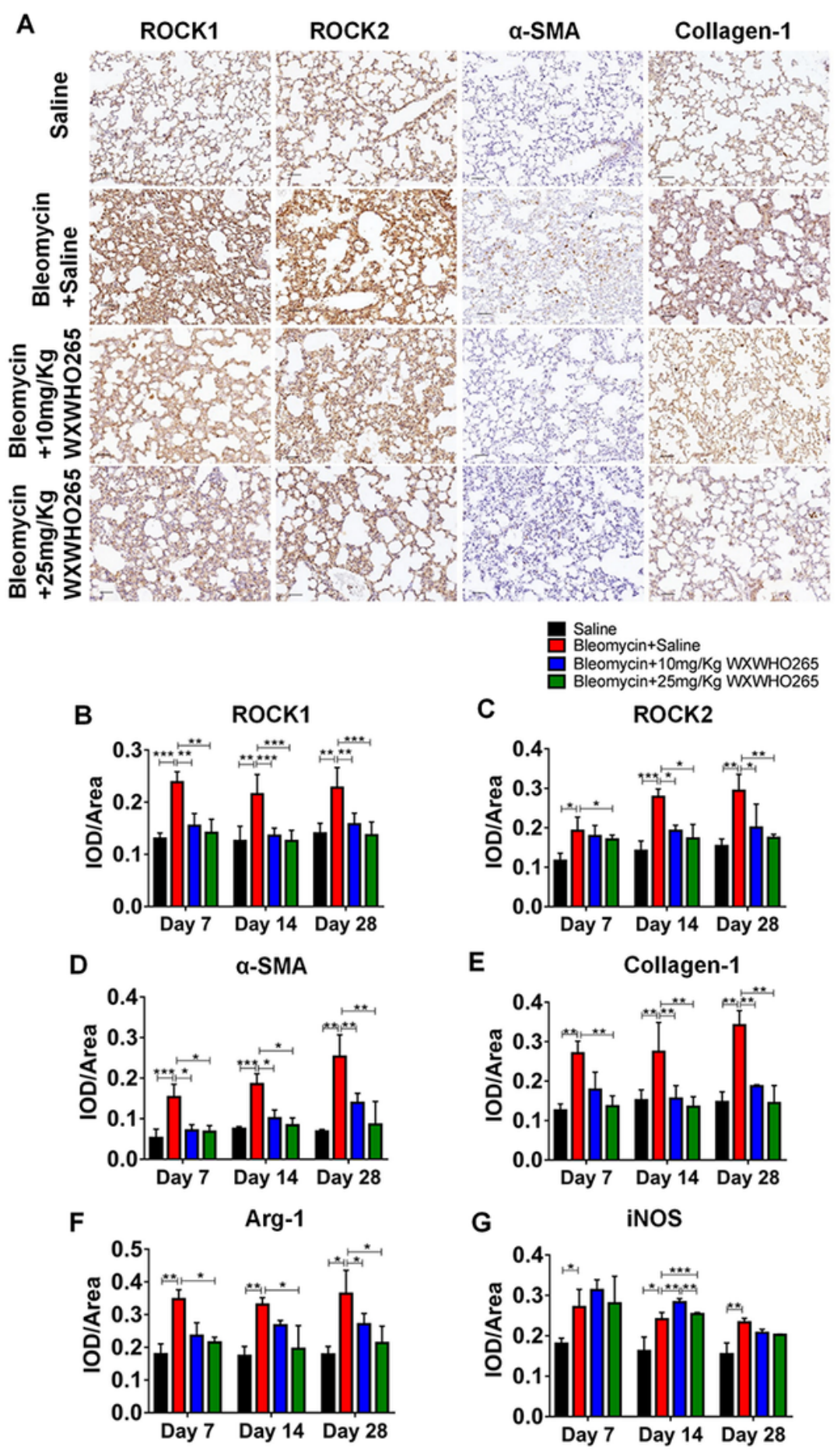

Figure 2

ROCK inhibitor (WXWHO265) ameliorated pulmonary fibrosis and decreased the expression of ROCK1, ROCK2, a-SMA and Collagen-1 in lung tissue. Mice with intratracheal administration of bleomycin $(4 \mathrm{mg} / \mathrm{kg})$ or saline were treated with WXWHO265 (10 or $25 \mathrm{mg} / \mathrm{kg}$,) or saline. The mice were sacrificed at day 7, day 14 and day 28 and the lung specimens were harvested for immunohistochemical analysis and stained with ROCK1, ROCK2, SMA, and Collagen-1. (A) Representative pictures of IHC analysis on day 28 
were shown. Scale bar $=50 \mu \mathrm{m}$. (B-G) The mean optical density of ROCK1 (B), ROCK2 (C), a-SMA (D), Collagen-1 (E), Arg-1 (F) and iNOS (G) expression in the lung tissue at the day 7, day 14 and day 28. The data were analyzed by one-way ANOVA and shown as mean \pm SEM. ${ }^{*} p<0.05,{ }^{* *} p<0.01,{ }^{* * *} p<0.001$.
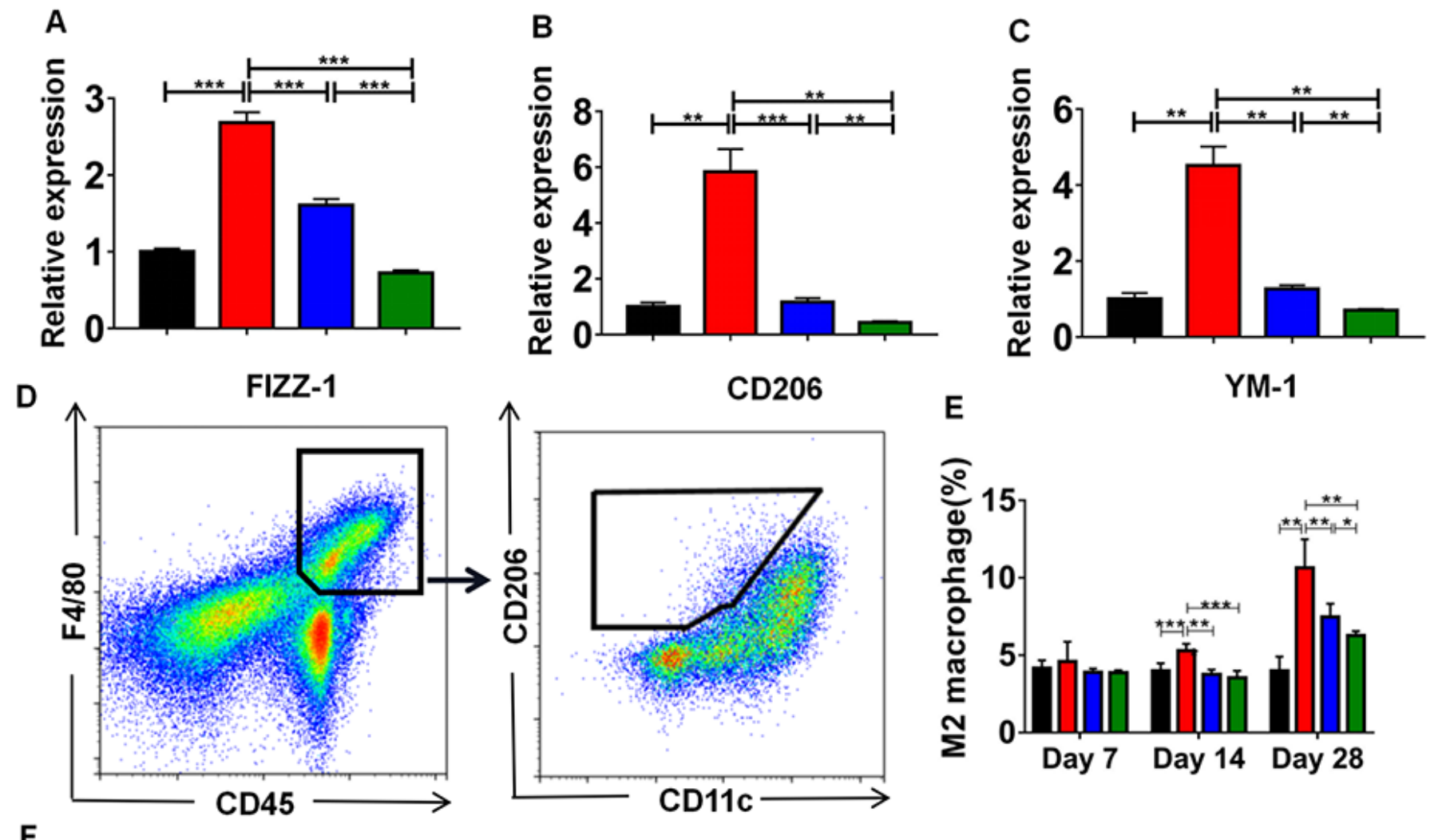

F
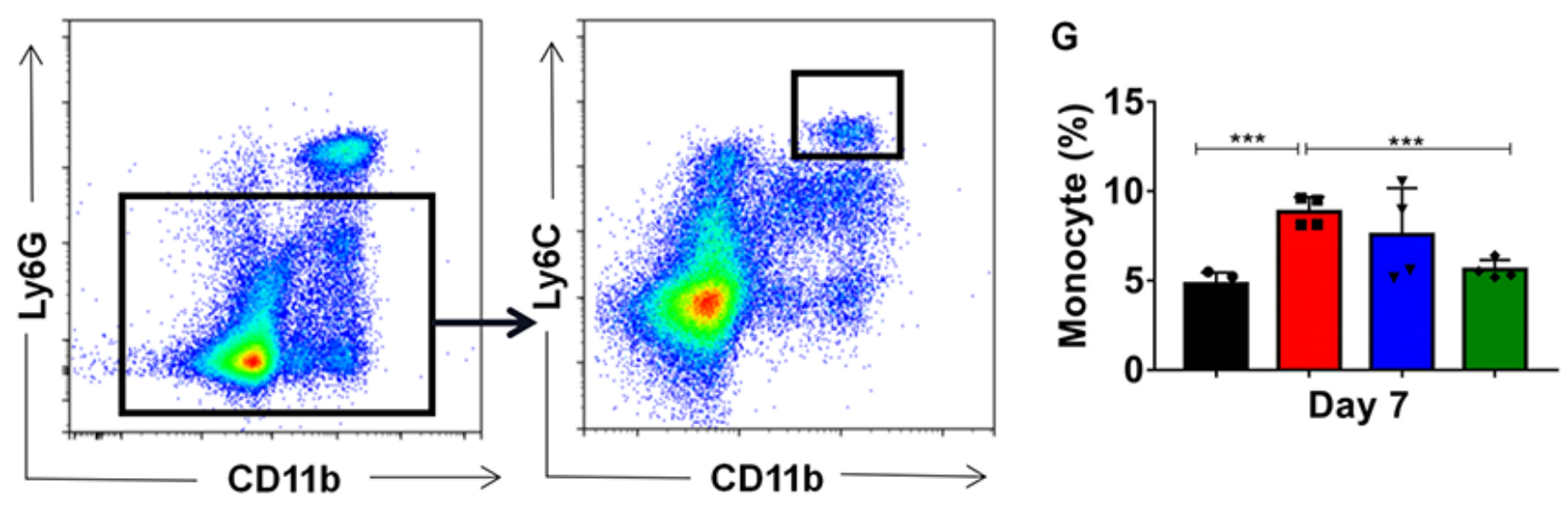

Figure 3

The infiltration of M2 macrophages and monocytes in bleomycin-induced PF model. After the establishment of bleomycin-induced PF model, mice were treated with WXWHO265. On day 7, day 14, day 28 , the lung tissues and blood were collected and digested for further analyses. (A-C) The relative mRNA expression of FIZZ-1 (A), CD206 (B) and YM-1 (C) in lung tissue on day 28. The expression of GAPDH 
was used as control. (D-E) The infiltration of M2 macrophages (CD45+F4/80+CD206+CD11c-) in lung tissue and monocytes (CD45+CD11b+Ly6G-Ly6Chigh) in blood was detected by flow cytometry. Flow cytometric quantification of infiltrated M2 macrophages (D) and monocytes (E) were shown. The data were analyzed by one-way ANOVA and shown as mean \pm SEM. ${ }^{*} p<0.05,{ }^{* *} p<0.01,{ }^{* * *} p<0.001$.
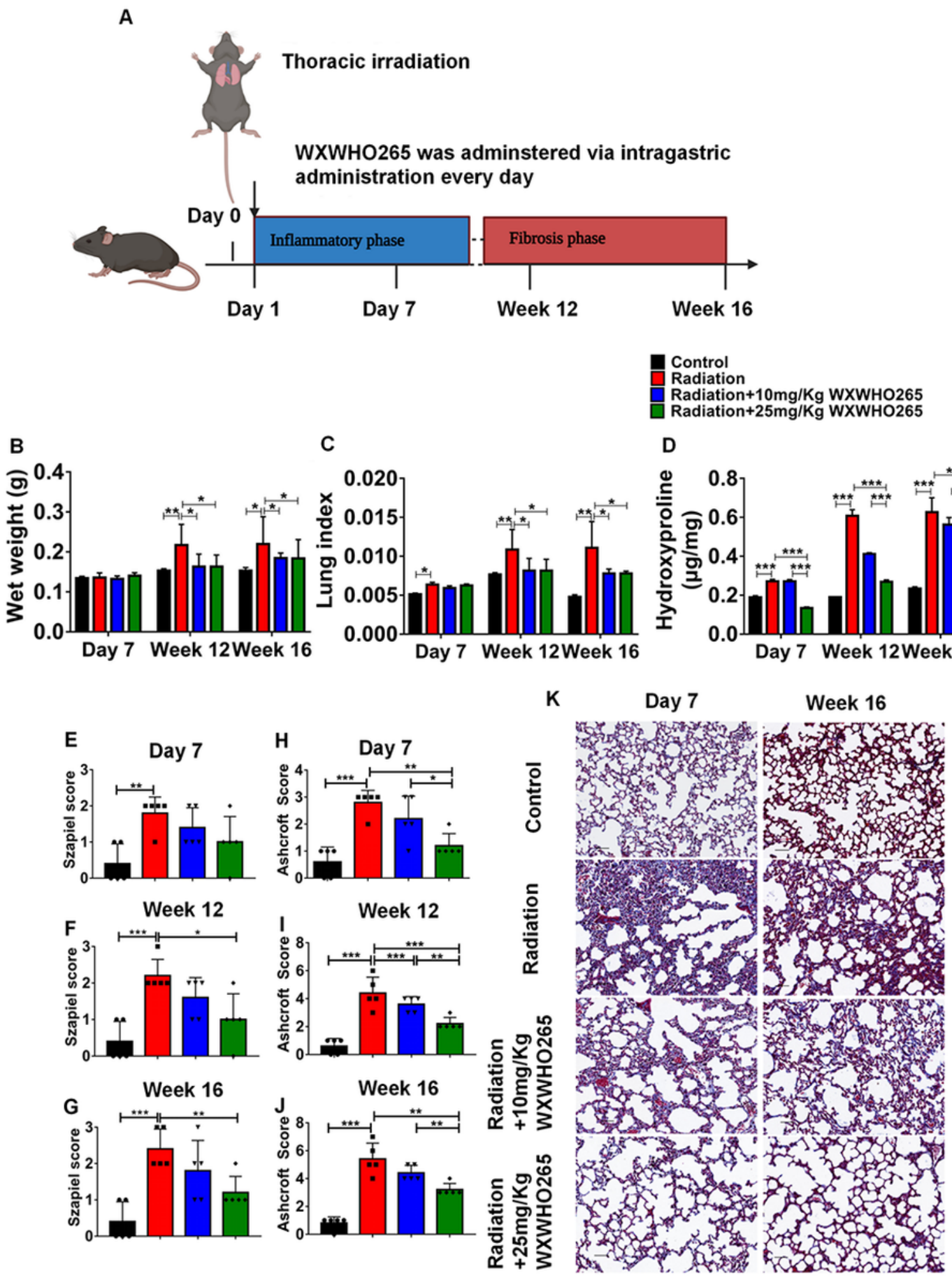

Figure 4 
The treatment effects of ROCK inhibitor (WXWHO265) in radiation-induced lung fibrosis model. (A) Schematic representation of the experimental protocol for model induction, treatment, and endpoints in the radiation-induced lung fibrosis mice model. For radiation induced model, mice were exposed to a single dose of $18 \mathrm{~Gy}$ irradiation on the bilateral thorax. $\mathrm{n}=6-7$. At the day after modeling, the ROCK inhibitor WXWHO265 was administered at $10 \mathrm{mg} / \mathrm{kg}$ or $25 \mathrm{mg} / \mathrm{kg}$ by intragastric injection every day. At the endpoint, the lung tissue and blood were collected for further experiments. (B-D) After bilateral thorax irradiation, the mice were treated with WXWHO265 or blank. The wet weight of lung (B), lung index (C), and hydroxyproline expression in lung (D) at day 7, week 12, week 16 are shown. Lung index was referred to lung/body weight ratio. (E-G) The inflammation changes in the lungs were quantified with a numerical inflammation score (Szapiel score) histopathologically. $(\mathrm{H}-\mathrm{J})$ The fibrotic changes in the lungs were quantified with a numerical fibrotic score (Ashcroft score) histopathologically. (K) Masson staining of mice lung tissues at day 7 and week 16 . Representative pictures are shown, scale bar $=50 \mu \mathrm{m}$. The data were analyzed by one-way ANOVA and shown as mean \pm SEM. ${ }^{*} p<0.05,{ }^{* *} p<0.01,{ }^{* \star *} p<0.001$. 
Control

Radiation

Radiation+10mg/Kg WXWHO265

Radiation $+25 \mathrm{mg} / \mathrm{Kg}$ WXWHO265
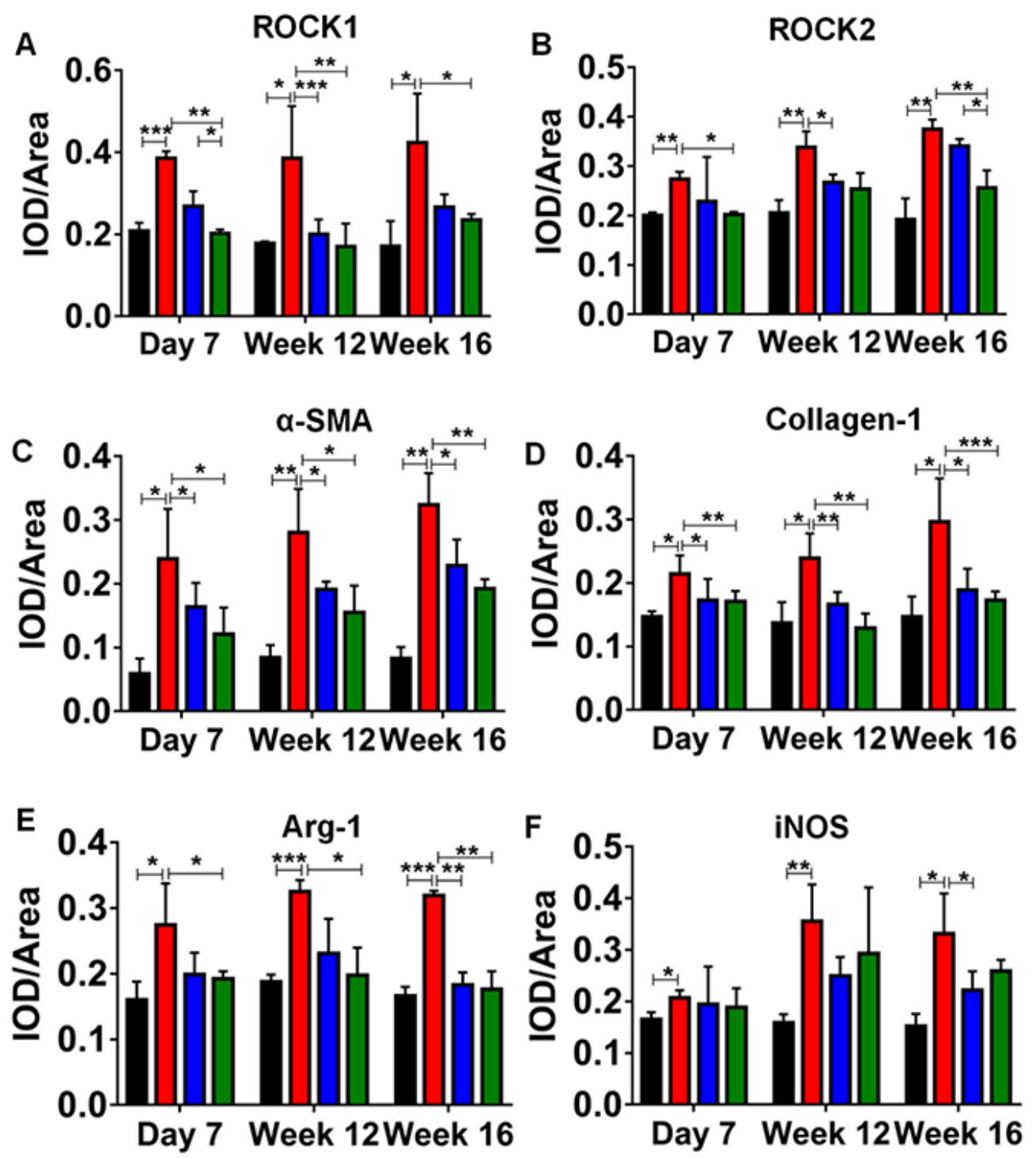

Figure 5

ROCK inhibitor (WXWHO265) ameliorated radiation-induced pulmonary fibrosis and decreased the expression of ROCK1, ROCK2, a-SMA, Collagen-1, Arg-1 and iNOS in lung tissue. After exposure to 18Gy bilateral thoracic irradiation and treated with WXWHO265, the mice were sacrificed at day 7, week 12 and week 16 and the lung specimens were harvested for immunohistochemical analysis. (A-F) IHC staining for the mean optical density of ROCK1(A), ROCK2 (B), a-SMA (C), Collagen-1 (D), Arg-1 (E) and iNOS (F) expression in the lung tissue at day 7 , week 12 , and week 16 . The data were analyzed by one-way ANOVA and shown as mean \pm SEM, ${ }^{*} p<0.05,{ }^{* *} p<0.01,{ }^{* * *} p<0.001$. 

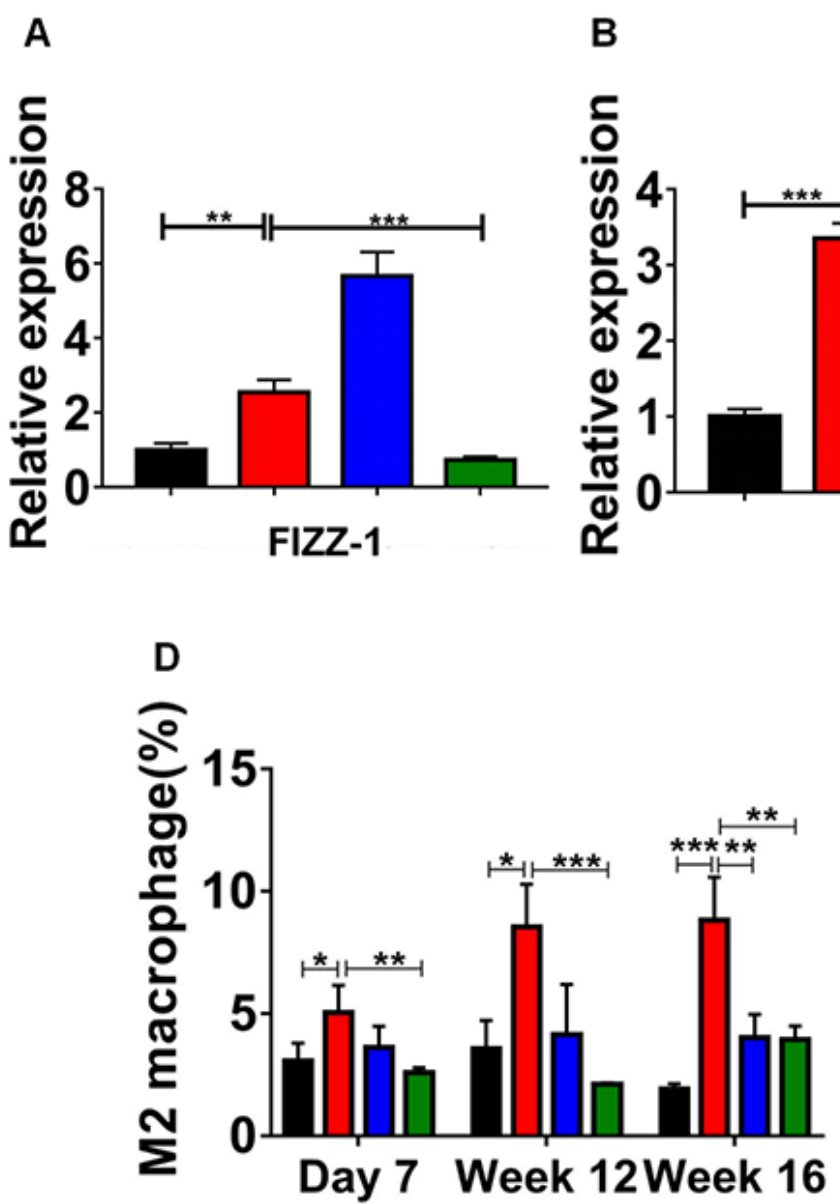

B

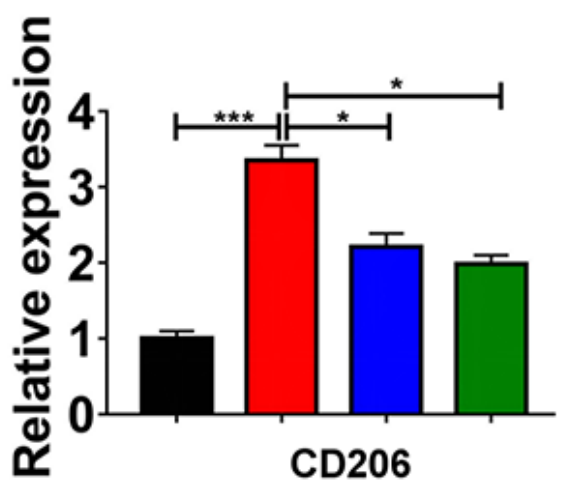

E

C
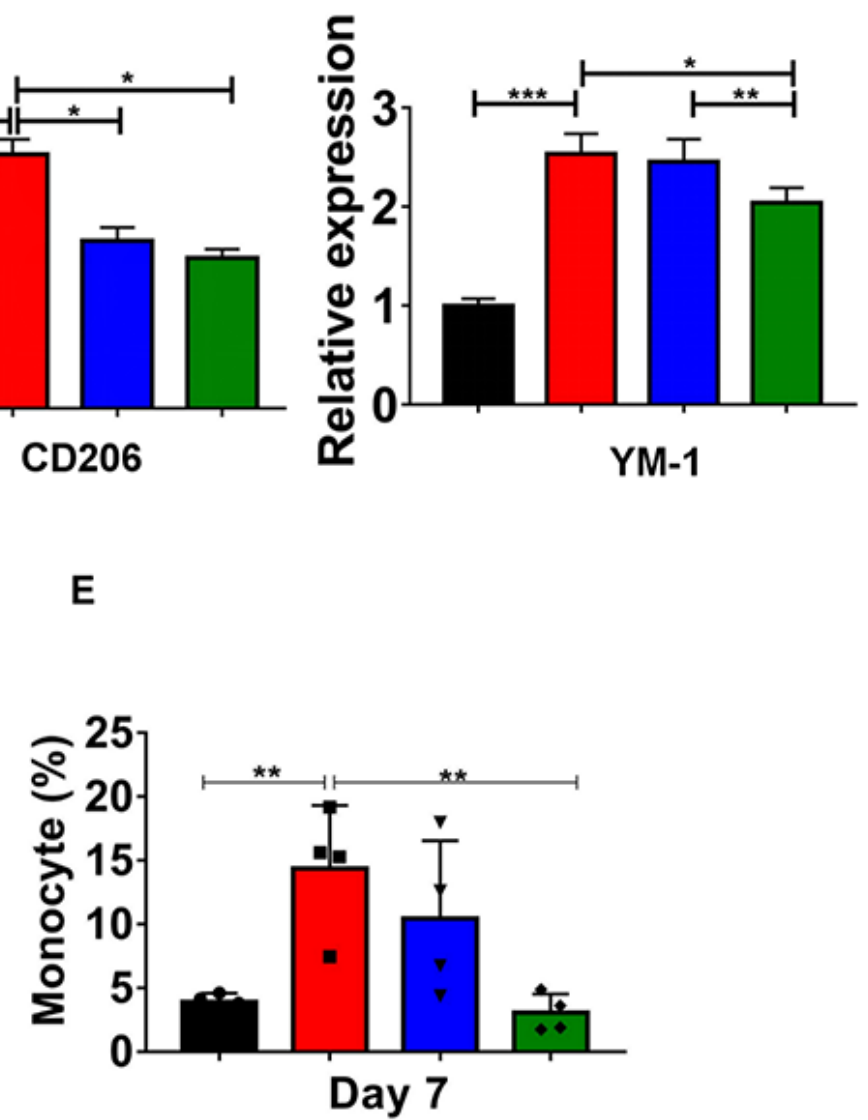

Figure 6

The infiltration of M2 macrophages and monocytes in the lung tissue of radiation-induced model treated with WXWH0265. The lung tissues of experimental groups were collected at day 7, week 12, and week 16. (A-C) The relative mRNA expression of FIZZ-1 (A), CD206 (B) and YM-1 (C) in lung tissue of radiationinduced model at week 16. (D-E) The infiltration of M2 macrophages (CD45+F4/80+CD206+CD11C-) in lung tissue (D) and monocyte (CD45+CD11b+Ly6G-Ly6Chigh) in blood (E) was detected by flow cytometry. ${ }^{*} p<0.05,{ }^{*} p<0.01,{ }^{* *} p<0.001$. Data were shown as mean $\pm S E M,{ }^{*} p<0.05,{ }^{* *} p<0.01$, $\star \star * p<0.001$. 
A
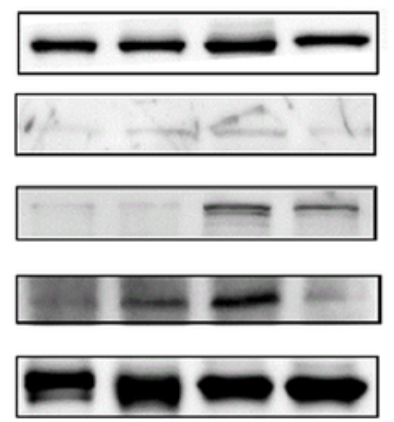

WH WL BLM Sal

C
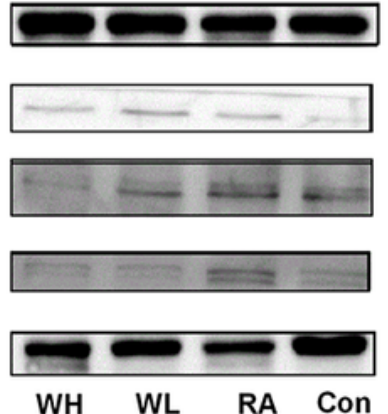

STAT3

p-STAT3

ROCK2

ROCK1

$\beta$-Tumblin

STAT3

p-STAT3

ROCK2

ROCK1

$\beta$-Tumblin
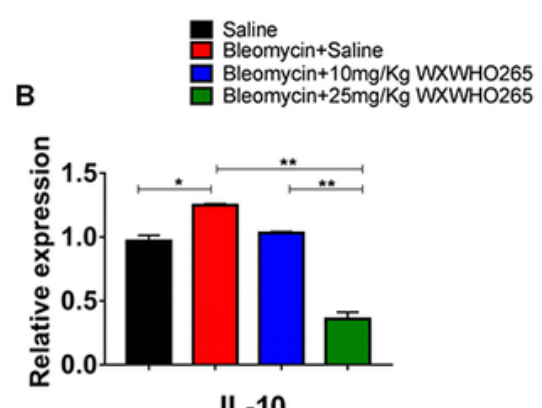

IL-10

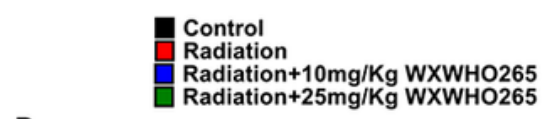

D

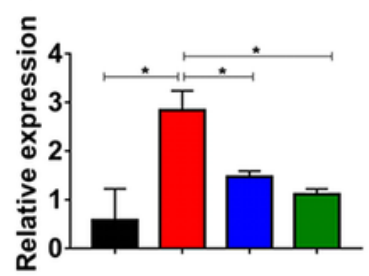

IL-10

E

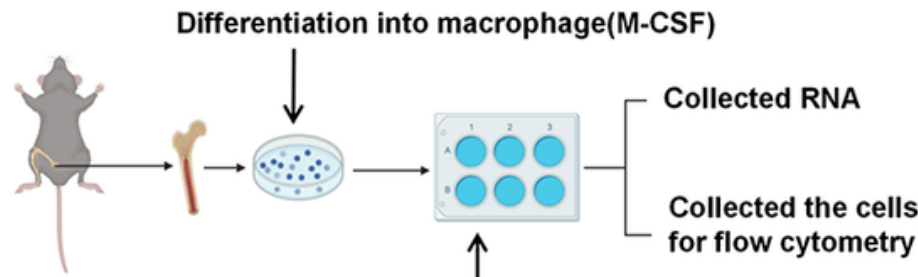

IL-4 was added. After 2 hour, WXWHO265 was added and stimulated for $\mathbf{4 8}$ hour
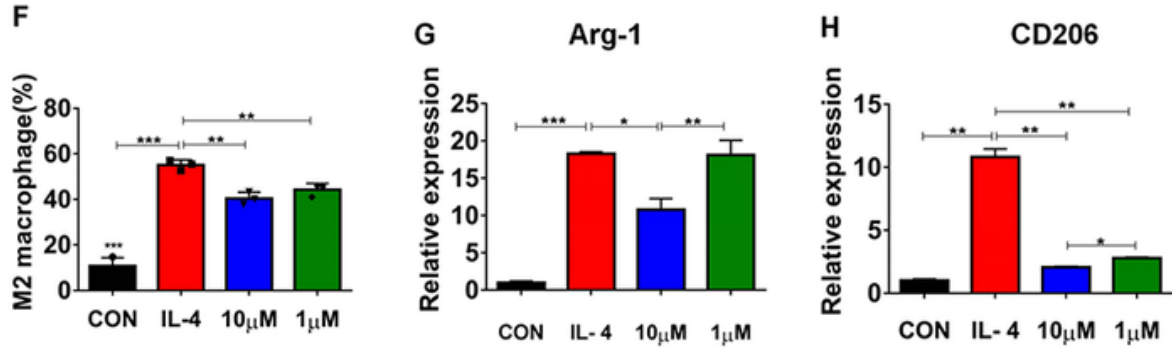

\section{Figure 7}

The mechanism of ROCK inhibitor regulating the polarization of M2 macrophage. After intratracheal instillation of bleomycin, mice were treated with WXWHO265 and scarified at day 28. The lung tissues were collected and digested for further analyses. (A) Western blot analysis of the expression of ROCK1, ROCK2, p-STAT3, STAT3 in bleomycin-induced mice model lung tissue. Saline: Sal; Bleomycin + saline: BLM; Bleomycin+10mg/Kg WXWH0265: WL; Bleomycin+25mg/Kg WXWHO265: WH. (B) The relative 
mRNA expression of IL-10 in the lung on day 28. After exposed to irradiation at bilateral thorax, mice were treated with WXWHO265 and sacrificed at week 16. (C) The Western blot analysis of the expression of ROCK1, ROCK2, p-STAT3, STAT3 radiation-induced mice model in lung tissue. Control: Con; Radiation: RA; Radiation+10mg/Kg WXWHO265: WL; Radiation+25mg/kg WXWHO265:WH. (D) The relative mRNA expression of IL-10 in the lung. (E-H) Bone marrow derived macrophages (BMDMs) were extracted from the femurs of untreated wild type mice and IL-4 was added at $20 \mathrm{ng} / \mathrm{ml}$ in the culture medium. After cultured for 2 hours, $10 \mu \mathrm{M}$ and $1 \mu \mathrm{M}$ of WXWHO265 was added into the medium for another 48 hours and then the BMDMs were harvested for further analyses. The experimental design protocol (E) was shown. The proportion of M2 macrophage (CD45+CD11b+F4/80+CD206+) was analyzed by flow cytometry $(F)$. The relative mRNA expressions of Arg-1 $(\mathrm{G})$ and CD206 $(\mathrm{H})$ in the BMDM were quantified by qRT-PCR. Data were shown as mean \pm SEM, ${ }^{*} p<0.05,{ }^{\star *} p<0.01,{ }^{\star \star *} \mathrm{p}<0.001$.

\section{Supplementary Files}

This is a list of supplementary files associated with this preprint. Click to download.

- SupplementaryTable1.docx 